\title{
Asymptotic Behavior of a Discrete Maturity Structured System of Hematopoietic Stem Cell Dynamics with Several Delays
}

\author{
M. Adimy ${ }^{a, b}$, F. Crauste ${ }^{c 1}$ and A. El Abdllaoui ${ }^{a}$ \\ ${ }^{a}$ Laboratoire de Mathématiques Appliquées CNRS UMR 5142 \\ Université de Pau et des Pays de l'Adour, 64000 Pau, France \\ ${ }^{b}$ ANUBIS Team, INRIA Futurs \\ ${ }^{c}$ Université de Lyon, Université Lyon1, CNRS UMR 5208 Institut Camille Jordan \\ F - 69200 Villeurbanne, France
}

\begin{abstract}
We propose and analyze a mathematical model of hematopoietic stem cell dynamics. This model takes into account a finite number of stages in blood production, characterized by cell maturity levels, which enhance the difference, in the hematopoiesis process, between dividing cells that differentiate (by going to the next stage) and dividing cells that keep the same maturity level (by staying in the same stage). It is described by a system of $n$ nonlinear differential equations with $n$ delays. We study some fundamental properties of the solutions, such as boundedness and positivity, and we investigate the existence of steady states. We determine some conditions for the local asymptotic stability of the trivial steady state, and obtain a sufficient condition for its global asymptotic stability by using a Lyapunov functional. Then we prove the instability of axial steady states. We study the asymptotic behavior of the unique positive steady state and obtain the existence of a stability area depending on all the time delays. We give a numerical illustration of this result for a system of four equations.
\end{abstract}

Key words: Hematopoiesis modelling, system of delay equations, global and local asymptotic stability, Lyapunov functional.

AMS subject classification: 34K20, 92C37, 34C23, 34D20, 34K99

\footnotetext{
${ }^{1}$ Corresponding author. Email: crauste@math.univ-lyon1.fr
} 


\section{Introduction}

All blood cells, that is red blood cells, white cells, and platelets, are produced in the bone marrow by so-called hematopoietic stem cells. They are pluripotent stem cells, with selfrenewal and differentiation abilities. Throughout divisions, hematopoietic stem cells produce an increasing number of differentiated cells (with some well known stages, such as progenitor cells, precursor cells) until the process leads to mature cells. These latter finally reach the blood stream and become blood cells.

Mathematical modelling of hematopoietic stem cells' dynamics has been introduced by Mackey [19], in 1978. The model of Mackey, based on earlier works by Lajtha [18] and Burns and Tannock [9], is an uncoupled system of two nonlinear delay differential equations, which considers a stem cell population divided in two compartments, a proliferating and a nonproliferating phase. The time delay corresponds to the proliferating phase duration. This model has been studied and improved by many authors (see Mackey and Rudnicki [20, 21], Dyson et al. [10, 11, 12], Pujo-Menjouet and Mackey [24], Adimy et al. [1, 2, 3, 4], and the references therein).

In each attempt to describe the dynamics of the hematopoietic stem cell population (see the above references), the authors only took into account a system of two differential equations to describe the whole process of differentiation. In this work, we propose a model which consider a finite number of stages during the production of blood cells, corresponding to different maturity levels (see Figure 1). This idea can be found in Bernard et al. [8], where the authors used a (discrete) maturity variable in their modelling, but did not take into account the self-renewal capacity of hematopoietic stem cells which allows some cells to stay in the same maturity compartment as their mother, and do not commit them to go further in the differentiation process at each division.

We consider a system of $n$ nonlinear differential equations, with delays, based on the model of Mackey [19] — thus each cell can be either in a proliferating phase, where it performs a series of process, such as growth, DNA synthesis, which ends with the cell division, or in a nonproliferating phase, where it can stay its entire life. Nonproliferating cells can reach the proliferating phase whenever during their life with a nonlinear rate $\beta$-, in which a part of the cell population in a given compartment leaves it at division to go to the next one, and the other part stays in the compartment. The proportion of cells that leave is supposed to be constant in each compartment.

In the next section, we present the model and some properties of the solutions. Then, we investigate the existence of steady states in Section 3. The linearization of the system about one of its steady states is performed in Section 4 and leads to the study of the global asymptotic stability of the trivial equilibrium in Section 5 and the local asymptotic stability of the unique positive steady state in Section 6. In this last section, we prove the existence of a stability area for the positive steady state, depending on all the time delays. 


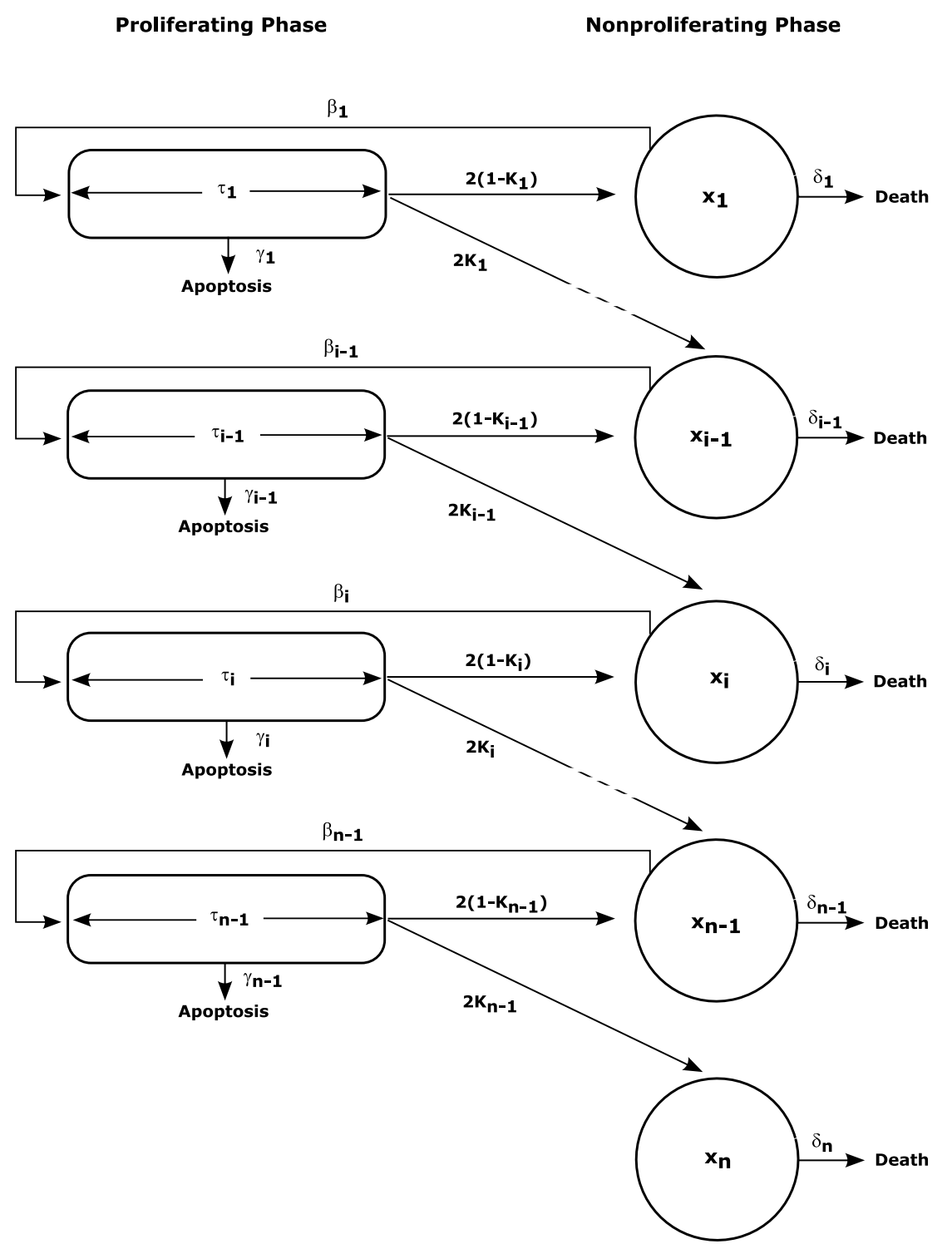

Figure 1: The hematopoietic stem cell (HSC) population is divided in proliferating (on the left hand side) and nonproliferating (on the right hand side) cells. Moreover, the HSC population is distributed within different compartments, depending on their maturity levels. Here, $n$ compartments are considered, the first one being related to pluripotent HSC, and the last one to mature cells, which do not proliferate anymore. The nonproliferating cell population in the $i$-th compartment is denoted by $x_{i}$. These cells can die with a rate $\delta_{i}$, and be introduced in the proliferating phase with a rate $\beta_{i}$. Proliferating cells have a time $\tau_{i}$ to perform the cell cycle and divide. They can die by apoptosis with a rate $\gamma_{i}$. The transfer from one maturity compartment to the next, say from the $i$-th compartment to the $i+1$-th one, is made at division. Cells always divide in two daughter cells, and a proportion $K_{i}$ of dividing cells go to the next nonproliferating phase, whereas others re-enter the same nonproliferating phase. 


\section{Mathematical Model}

Throughout this paper, we set

$$
I_{n}:=\{1, \ldots, n\}, \quad n \in \mathbb{N}, n \geq 3 .
$$

Let $x_{i}(t), i \in I_{n}$, denote the density of (nonproliferating) hematopoietic stem cells in the $i$-th compartment at time $t$. Then $x_{i}$ satisfies (see Figure 1)

$$
\begin{aligned}
\frac{d x_{i}}{d t}(t)= & -\left[\delta_{i}+\beta_{i}\left(x_{i}(t)\right)\right] x_{i}(t)+2\left(1-K_{i}\right) e^{-\gamma_{i} \tau_{i}} \beta_{i}\left(x_{i}\left(t-\tau_{i}\right)\right) x_{i}\left(t-\tau_{i}\right) \\
& +2 K_{i-1} e^{-\gamma_{i-1} \tau_{i-1}} \beta_{i-1}\left(x_{i-1}\left(t-\tau_{i-1}\right)\right) x_{i-1}\left(t-\tau_{i-1}\right)
\end{aligned}
$$

where, for $i$ fixed, the mortality rates in the nonproliferating phase $\delta_{i}$ and in the proliferating one $\gamma_{i}$, the proliferating phase durations $\tau_{i}$, and the proportion of cells that leave the stage $i$ to the stage $i+1, K_{i}$, are constant, and the introduction rates $\beta_{i}:[0,+\infty) \rightarrow \mathbb{R}$ are continuous functions. We assume that, for $i \in I_{n-1}$,

$$
\begin{array}{ll}
\text { (H1) } & 0<\delta_{i} \leq \delta_{i+1}, \quad 0<\gamma_{i} \leq \gamma_{i+1}, \quad \text { and } \quad 0 \leq \tau_{i} \leq \tau_{i+1} \text {; } \\
\text { (H2) } & 0:=K_{0}<K_{i} \leq K_{i+1}<1 .
\end{array}
$$

From [17, 22], it seems that the proliferating phase duration $\tau_{i}$ is an increasing function of cell maturity (immature cells dividing faster than mature cells), as well as mortality rates, justifying hypothesis (H1). Moreover, as cells become more and more mature, they lose their self-renewal capacity so the differentiation rate $K_{i}$ increases (hypothesis (H2)) and becomes closer to one.

Moreover, we suppose that the functions $\beta_{i}$ satisfy

$$
0<\beta_{i+1}(0) \leq \beta_{i}(0) \quad \text { for } i \in I_{n-2}, \quad \text { and } \quad \beta_{n} \equiv 0 \text {; }
$$

(H4) for $i \in I_{n-1}, \beta_{i}$ is a positive and decreasing function such that

$$
\lim _{x \rightarrow+\infty} \beta_{i}(x)=0 .
$$

Remark 1. Note that indeed $\gamma_{0}, \tau_{0}, \beta_{0}$ and $x_{0}$ do not play any role in (2.1), since $K_{0}=0$, as well as $K_{n}, \gamma_{n}$ and $\tau_{n}$, since $\beta_{n} \equiv 0$. 
Hence, system (2.1) must be read as

$$
\left\{\begin{aligned}
\frac{d x_{1}}{d t}(t)= & -\left[\delta_{1}+\beta_{1}\left(x_{1}(t)\right)\right] x_{1}(t) \\
& +2\left(1-K_{1}\right) e^{-\gamma_{1} \tau_{1}} \beta_{1}\left(x_{1}\left(t-\tau_{1}\right)\right) x_{1}\left(t-\tau_{1}\right) \\
\frac{d x_{i}}{d t}(t)= & -\left[\delta_{i}+\beta_{i}\left(x_{i}(t)\right)\right] x_{i}(t) \\
& +2\left(1-K_{i}\right) e^{-\gamma_{i} \tau_{i}} \beta_{i}\left(x_{i}\left(t-\tau_{i}\right)\right) x_{i}\left(t-\tau_{i}\right) \\
& +2 K_{i-1} e^{-\gamma_{i-1} \tau_{i-1}} \beta_{i-1}\left(x_{i-1}\left(t-\tau_{i-1}\right)\right) x_{i-1}\left(t-\tau_{i-1}\right), \quad i=2, \ldots, n-1, \\
\frac{d x_{n}}{d t}(t)= & -\delta_{n} x_{n}(t) \\
& +2 K_{n-1} e^{-\gamma_{n-1} \tau_{n-1}} \beta_{n-1}\left(x_{n-1}\left(t-\tau_{n-1}\right)\right) x_{n-1}\left(t-\tau_{n-1}\right)
\end{aligned}\right.
$$

From Hale and Verduyn Lunel [14], system (2.1) admits a unique solution for each continuous initial condition. The next proposition states and proves positivity and boundedness of solutions of system (2.1).

Proposition 2. The solutions of system (2.1) associated with nonnegative initial conditions are nonnegative and bounded.

Proof. We first show that solutions of (2.1) are nonnegative.

First notice that $x_{1}$ is nonnegative. Indeed, assume that there exists $T>0$ such that $x_{1}(t)>0$ for $t<T$ and $x_{1}(T)=0$. Then, from (2.1), using (H2) and (H4),

$$
\frac{d x_{1}}{d t}(T)=2\left(1-K_{1}\right) e^{-\gamma_{1} \tau_{1}} \beta_{1}\left(x_{1}\left(T-\tau_{1}\right)\right) x_{1}\left(T-\tau_{1}\right)>0 .
$$

Consequently, $x_{1}$ is nonnegative.

Now assume that $x_{i}, i \geq 1$, is nonnegative and let us show that $x_{i+1}$ is nonnegative. Suppose that there exists $T>0$ such that $x_{i+1}(t)>0$ for $t<T$ and $x_{i+1}(T)=0$. Then, from (2.1), using (H2) and (H4),

$$
\begin{aligned}
\frac{d x_{i+1}}{d t}(T)= & 2\left(1-K_{i+1}\right) e^{-\gamma_{i+1} \tau_{i+1}} \beta_{i+1}\left(x_{i+1}\left(T-\tau_{i+1}\right)\right) x_{i+1}\left(T-\tau_{i+1}\right) \\
& +2 K_{i} e^{-\gamma_{i} \tau_{i}} \beta_{i}\left(x_{i}\left(T-\tau_{i}\right)\right) x_{i}\left(T-\tau_{i}\right)>0
\end{aligned}
$$

So the conclusion follows.

Now, let us prove that the solutions of (2.1) are bounded. For $i \in I_{n}$, define the functions $f_{i}, g_{i-1}:[0,+\infty) \rightarrow \mathbb{R}^{+}$by

$$
f_{i}(y)=2\left(1-K_{i}\right) e^{-\gamma_{i} \tau_{i}} \beta_{i}(y) \quad \text { and } \quad g_{i-1}(y)=2 K_{i-1} e^{-\gamma_{i-1} \tau_{i-1}} \beta_{i-1}(y) .
$$


From $(\mathbf{H} 4)$, the functions $f_{i}$ and $g_{i-1}$ are decreasing and tend to 0 when $y$ tends to infinity (except the functions $g_{0}$ and $f_{n}$, that satisfy $f_{n} \equiv g_{0} \equiv 0$ ). Therefore we can find a real number $\chi_{0}^{i} \geq 0$ such that

$$
f_{i}(y)<\frac{\delta_{i}}{2} \quad \text { and } \quad g_{i-1}(y)<\frac{\delta_{i}}{2} \quad \text { for } y>\chi_{0}^{i} .
$$

Define then the quantity

$$
\chi_{1}^{i}:=\frac{2 \chi_{0}^{i}}{\delta_{i}}\left(f_{i}(0)+g_{i-1}(0)\right) .
$$

Let $x>\chi_{1}^{i}$ and $y \in[0, x]$ be fixed. Then, using (2.3) and the fact that $f_{i}$ is decreasing, we obtain

$$
f_{i}(y) y \leq \begin{cases}f_{i}(0) \chi_{0}^{i}, & \text { if } y \leq \chi_{0}^{i} \\ \frac{\delta_{i}}{2} y, & \text { if } y>\chi_{0}^{i}\end{cases}
$$

Consequently, with the definition of $\chi_{1}^{i}$, we deduce

$$
f_{i}(y) y \leq \frac{\delta_{i}}{2} x
$$

The same reasoning yields

$$
g_{i-1}(y) y \leq \frac{\delta_{i}}{2} x
$$

Thus, for all $x>\chi_{1}^{i}$, we have

$$
\max _{0 \leq y, z \leq x}\left(f_{i}(y) y+g_{i-1}(z) z\right) \leq \delta_{i} x .
$$

Assume now that $x_{1}$ is unbounded. Then one can find $t_{0}>0$ such that

$$
x_{1}(t) \leq x_{1}\left(t_{0}\right) \text { for } t \in\left[t_{0}-\tau_{1}, t_{0}\right], \quad \text { and } \quad x_{1}\left(t_{0}\right)>\chi_{1}^{1} .
$$

Then, from (2.1) and using (2.4), we have

$$
\begin{aligned}
\frac{d x_{1}}{d t}\left(t_{0}\right) & =-\left[\delta_{1}+\beta_{1}\left(x_{1}\left(t_{0}\right)\right)\right] x_{1}\left(t_{0}\right)+f_{1}\left(x_{1}\left(t_{0}-\tau_{1}\right)\right) x_{1}\left(t_{0}-\tau_{1}\right) \\
& \leq-\left[\delta_{1}+\beta_{1}\left(x_{1}\left(t_{0}\right)\right)\right] x_{1}\left(t_{0}\right)+\delta_{1} x_{1}\left(t_{0}\right) \\
& =-\beta_{1}\left(x_{1}\left(t_{0}\right)\right) x_{1}\left(t_{0}\right) \\
& <0
\end{aligned}
$$

This leads to a contradiction, so we deduce that $x_{1}$ is bounded.

Assume now that $x_{i}, i \geq 1$, is bounded, and let us show that $x_{i+1}$ is bounded. Suppose, by contradiction, that $x_{i+1}$ is unbounded. Then one can find $t_{0}>0$ such that

$$
\begin{gathered}
x_{i+1}(t) \leq x_{i+1}\left(t_{0}\right) \quad \text { and } \quad x_{i}(t) \leq x_{i+1}\left(t_{0}\right), \quad \text { for } t \in\left[t_{0}-\tau_{i+1}, t_{0}\right], \\
\text { and } \quad x_{i+1}\left(t_{0}\right)>\chi_{1}^{i+1} .
\end{gathered}
$$


Then, from (2.1) and using (2.4), the same reasoning than the one used for $x_{1}$ leads to

$$
\begin{aligned}
\frac{d x_{i+1}}{d t}\left(t_{0}\right)= & -\left[\delta_{i+1}+\beta_{i+1}\left(x_{i+1}\left(t_{0}\right)\right)\right] x_{i+1}\left(t_{0}\right)+f_{i+1}\left(x_{i+1}\left(t_{0}-\tau_{i+1}\right)\right) x_{i+1}\left(t_{0}-\tau_{i+1}\right) \\
& +g_{i}\left(x_{i}\left(t_{0}-\tau_{i}\right)\right) x_{i}\left(t_{0}-\tau_{i}\right), \\
\leq & -\left[\delta_{i+1}+\beta_{i+1}\left(x_{i+1}\left(t_{0}\right)\right)\right] x_{i+1}\left(t_{0}\right)+\delta_{i+1} x_{i+1}\left(t_{0}\right), \\
< & 0 .
\end{aligned}
$$

This leads to a contradiction, so we deduce that, for all $i \in I_{n}, x_{i}$ is bounded.

We investigate, in the next section, the existence of steady states for system (2.1).

\section{$3 \quad$ Existence of Steady States}

We now focus on the existence of steady states for system $(2.1)$. Let $\bar{E}=\left(\bar{x}_{1}, \ldots, \bar{x}_{n}\right)^{\mathrm{T}}$ be a steady state of (2.1). Then $\bar{E}$ satisfies

$$
\frac{d \bar{x}_{1}}{d t}=\cdots=\frac{d \bar{x}_{n}}{d t}=0
$$

that is

$$
\left\{\begin{aligned}
{\left[\left(2\left(1-K_{1}\right) e^{-\gamma_{1} \tau_{1}}-1\right) \beta_{1}\left(\bar{x}_{1}\right)-\delta_{1}\right] \bar{x}_{1} } & =0, \\
\vdots & \\
2 K_{i-1} e^{-\gamma_{i-1} \tau_{i-1}} \beta_{i-1}\left(\bar{x}_{i-1}\right) \bar{x}_{i-1} & \\
+\left[\left(2\left(1-K_{i}\right) e^{-\gamma_{i} \tau_{i}}-1\right) \beta_{i}\left(\bar{x}_{i}\right)-\delta_{i}\right] \bar{x}_{i} & =0, \quad \text { for } i=2, \ldots, n-1, \\
\vdots & \\
2 K_{n-1} e^{-\gamma_{n-1} \tau_{n-1}} \beta_{n-1}\left(\bar{x}_{n-1}\right) \bar{x}_{n-1}-\delta_{n} \bar{x}_{n} & =0 .
\end{aligned}\right.
$$

System (3.5) is equivalent to

$$
\left(\begin{array}{ccccc}
\xi_{1}\left(\bar{x}_{1}\right) & 0 & \ldots & \ldots & 0 \\
g_{1}\left(\bar{x}_{1}\right) & \xi_{2}\left(\bar{x}_{2}\right) & 0 & \ldots & 0 \\
0 & g_{2}\left(\bar{x}_{2}\right) & \xi_{3}\left(\bar{x}_{3}\right) & 0 & \vdots \\
\vdots & & & & \vdots \\
0 & \ldots & 0 & g_{n-1}\left(\bar{x}_{n-1}\right) & \xi_{n}\left(\bar{x}_{n}\right)
\end{array}\right)\left(\begin{array}{c}
\bar{x}_{1} \\
\\
\vdots \\
\bar{x}_{n}
\end{array}\right)=\left(\begin{array}{c}
0 \\
\\
\vdots \\
0
\end{array}\right)
$$

where the functions $g_{i}$ are defined in (2.2) and

$$
\xi_{i}(x):=\left(2\left(1-K_{i}\right) e^{-\gamma_{i} \tau_{i}}-1\right) \beta_{i}(x)-\delta_{i}, \quad \text { for } i \in I_{n},
$$

with $\xi_{n}(x)=-\delta_{n}$. 
Remark 3. Even though we do not write it explicitly, the functions $\xi_{i}$ and $g_{i}$ depend on $\tau_{i}$.

System (3.6) can be writen as

$$
A(\bar{E}) \bar{E}=0,
$$

where $A(\bar{E})$ is the $(n \times n)$ matrix given in (3.6).

It is noticeable that $E^{0}=(0, \ldots, 0)^{\mathrm{T}}$ is always an equilibrium point. Moreover, if the following condition holds

$$
\left(2\left(1-K_{1}\right) e^{-\gamma_{1} \tau_{1}}-1\right) \beta_{1}(0)<\delta_{1},
$$

then using (H1), (H2) and (H3), we obtain

$$
\left(2\left(1-K_{i}\right) e^{-\gamma_{i} \tau_{i}}-1\right) \beta_{i}\left(\bar{x}_{i}\right) \leq\left(2\left(1-K_{1}\right) e^{-\gamma_{1} \tau_{1}}-1\right) \beta_{1}(0)<\delta_{1} \leq \delta_{i}, \quad \text { for all } i \in I_{n} .
$$

So $\xi_{i}\left(\bar{x}_{i}\right)<0$ for all $i \in I_{n}$, and the matrix $A(\bar{E})$ in system (3.6) is invertible. Thus, under condition (3.8), $E^{0}$ is the unique equilibrium point.

Inequality (3.8) is satisfied, for example, if the mortality rates $\delta_{1}$ or $\gamma_{1}$ are large, or the introduction rate $\beta_{1}(0)$ is small. It describes a situation in which mortality takes advantage on cell renewal, for immature cells. From assumptions (H1), (H2) and (H3), this situation is then valid for all cells and the extinction becomes unavoidable.

In the sequel, we assume that

$$
\left(2\left(1-K_{1}\right) e^{-\gamma_{1} \tau_{1}}-1\right) \beta_{1}(0)>\delta_{1}
$$

Let $\bar{E}=\left(\bar{x}_{1}, \ldots, \bar{x}_{n}\right)^{\mathrm{T}} \neq E^{0}$ be a steady state of (2.1). From (3.6), $\bar{E}$ is a steady state if and only if

$$
\begin{aligned}
\xi_{1}\left(\bar{x}_{1}\right) \bar{x}_{1} & =0 \\
\xi_{i}\left(\bar{x}_{i}\right) \bar{x}_{i} & =-g_{i-1}\left(\bar{x}_{i-1}\right) \bar{x}_{i-1}, \quad \text { for all } i=2, \ldots, n
\end{aligned}
$$

It follows, from (3.10), that either $\bar{x}_{1}=0$ or $\xi_{1}\left(\bar{x}_{1}\right)=0$. Notice that, from (3.7) and (H4), the mapping $x \mapsto \xi_{i}(x), i \in I_{n-1}$ fixed, is decreasing for $x \geq 0$ and $\lim _{x \rightarrow+\infty} \xi_{i}(x)=-\delta_{i}$. Consequently, for $i \in I_{n-1}$, the equation $\xi_{i}(x)=0$ has a positive solution if and only if $\xi_{i}(0)>0$, and the solution is then unique. From $(3.9), \xi_{1}(0)>0$, so equation (3.10) has exactly two solutions, $\bar{x}_{1}=0$ and $\bar{x}_{1}=x_{1}^{*}>0$, unique solution of $\xi_{1}(x)=0$.

Now let $i \geq 2$ and $\bar{x}_{i-1}$ be fixed. We consider two cases: either $\bar{x}_{i-1}=0$ or $\bar{x}_{i-1}=x_{i-1}^{*}>$ 0 .

If $\bar{x}_{i-1}=0$, then $\bar{x}_{i}$ satisfies $\xi_{i}\left(\bar{x}_{i}\right) \bar{x}_{i}=0$. It follows that $\bar{x}_{i}=0$ or $\bar{x}_{i}=x_{i}^{*}>0$, where $\xi_{i}\left(x_{i}^{*}\right)=0$, provided that $\xi_{i}(0)>0$.

If $\bar{x}_{i-1}=x_{i-1}^{*}>0$, then $\bar{x}_{i}$ must satisfy $(3.11)$ so $\bar{x}_{i}$ is strictly positive and

$$
\xi_{i}\left(\bar{x}_{i}\right)=-\frac{g_{i-1}\left(x_{i-1}^{*}\right) x_{i-1}^{*}}{\bar{x}_{i}} .
$$


Since the mapping $x \mapsto-g_{i-1}\left(x_{i-1}^{*}\right) x_{i-1}^{*} / x$ is negative and increasing, with

$$
\lim _{x \rightarrow+\infty}-\frac{g_{i-1}\left(x_{i-1}^{*}\right) x_{i-1}^{*}}{x}=0,
$$

it follows that there exists a unique positive solution $\bar{x}_{i}=x_{i}^{*}$ to equation (3.11).

One can see that the situation is as follows: when $\bar{x}_{i-1}>0, \bar{x}_{i}$ is positive and uniquely determined as the solution of (3.11), whereas when $\bar{x}_{i-1}=0$ then $\bar{x}_{i}=0$ is always a solution, and a positive value of $\bar{x}_{i}$ may exist, provided that $\xi_{i}(0)>0$.

In order to determine the existence of equilibria according to the sign of $\xi_{i}(0)$, let us define

$$
i_{0}:=\min \left\{i \in I_{n} \mid \xi_{i}(0)<0\right\} .
$$

Since $\xi_{n}(0)=-\delta_{n}<0$, then $i_{0}$ is well-defined. Since, from $(\mathbf{H} 1),(\mathbf{H} 2)$ and $(\mathbf{H} 3)$, the mapping $i \mapsto \xi_{i}(0)$ is decreasing, then $\xi_{i}(0) \leq \xi_{i_{0}}(0)<0$, for all $i \geq i_{0}$. Consequently, $\xi_{i}(0)<0$ for all $i \geq i_{0}$. We state the following proposition.

\section{Proposition 4. Let}

$$
i_{0}:=\min \left\{i \in I_{n} \mid \xi_{i}(0)<0\right\} .
$$

Assume that $i_{0}=1$, that is $\xi_{1}(0)<0$. Then $E^{0}$ is the only steady state of system (2.1).

Assume that $i_{0} \geq 2$, that is $\xi_{1}(0)>0$. Then system (2.1) has $i_{0}$ equilibria. These equilibria, denoted by $E^{0}, E^{*}, E_{1}, \ldots, E_{i_{0}-2}$, are given by

$$
E^{0}=\left(\begin{array}{c}
0 \\
0 \\
\vdots \\
0
\end{array}\right), E^{*}=\left(\begin{array}{c}
x_{1}^{*} \\
x_{2}^{*} \\
\vdots \\
x_{n}^{*}
\end{array}\right), E_{1}=\left(\begin{array}{c}
0 \\
x_{2}^{*} \\
\vdots \\
x_{n}^{*}
\end{array}\right), \ldots, \text { and } E_{i_{0}-2}=\left(\begin{array}{c}
0 \\
\vdots \\
0 \\
x_{i_{0}-1}^{*} \\
\vdots \\
x_{n}^{*}
\end{array}\right) \text {, }
$$

where $x_{i}^{*}>0$ is the unique solution of the equation

$$
\left(2\left(1-K_{i}\right) e^{-\gamma_{i} \tau_{i}}-1\right) \beta_{i}(x)-\delta_{i}=0
$$

if $x_{i-1}^{*}=0$, and $x_{i}^{*}$ is the unique solution of the equation

$$
\left(2\left(1-K_{i}\right) e^{-\gamma_{i} \tau_{i}}-1\right) \beta_{i}(x)-\delta_{i}=-\frac{2}{x} K_{i-1} e^{-\gamma_{i-1} \tau_{i-1}} \beta_{i-1}\left(x_{i-1}^{*}\right) x_{i-1}^{*},
$$

if $x_{i-1}^{*}>0$.

Remark 5. It is easy to observe that $E_{j}$, with $1 \leq j \leq i_{0}-2$, is a vector whose the first $j$ components are zeros. 
Remark 6. Consider the equilibrium $E^{*}$ defined in Proposition 4. We want to stress the dependence of all $x_{i}^{*}$ on time delays $\tau_{i}$. First, it is easy to check that $x_{1}^{*}=x_{1}^{*}\left(\tau_{1}\right)$. Then, $x_{2}^{*}=x_{2}^{*}\left(x_{1}^{*}, \tau_{2}\right)$, that is $x_{2}^{*}=x_{2}^{*}\left(\tau_{1}, \tau_{2}\right)$, and so on for the other values $x_{i}^{*}=x_{i}^{*}\left(\tau_{1}, \ldots, \tau_{i}\right)$.

In the same way, one can notice that a nonzero component $x_{i}^{*}$ of an equilibrium $E_{j}$ (with $i>j)$, satisfies $x_{i}^{*}=x_{i}^{*}\left(\tau_{j+1}, \ldots, \tau_{i}\right)$.

In the next section, we linearize system (2.1) about one of its steady states to analyze its local asymptotic stability.

\section{Linearization}

Let $\bar{E}=\left(\bar{x}_{i}\right)_{i \in I_{n}}$ be a steady state of system (2.1). Assume $\beta_{i}, i \in I_{n-1}$, to be $C^{1}$ functions. The linearization of system (2.1) about $\bar{E}$ gives

$$
\left\{\begin{aligned}
\frac{d x_{1}}{d t}(t)= & -\left[\delta_{1}+\alpha_{1}\left(\bar{x}_{1}\right)\right] x_{1}(t)+2\left(1-K_{1}\right) e^{-\gamma_{1} \tau_{1}} \alpha_{1}\left(\bar{x}_{1}\right) x_{1}\left(t-\tau_{1}\right), \\
\frac{d x_{i}}{d t}(t)= & -\left[\delta_{i}+\alpha_{i}\left(\bar{x}_{i}\right)\right] x_{i}(t)+2\left(1-K_{i}\right) e^{-\gamma_{i} \tau_{i}} \alpha_{i}\left(\bar{x}_{i}\right) x_{i}\left(t-\tau_{i}\right) \\
& +2 K_{i-1} e^{-\gamma_{i-1} \tau_{i-1}} \alpha_{i-1}\left(\bar{x}_{i-1}\right) x_{i-1}\left(t-\tau_{i-1}\right), \quad \text { for } i=2, \ldots, n-1, \\
\frac{d x_{n}}{d t}(t)= & -\delta_{n} x_{n}(t)+2 K_{n-1} e^{-\gamma_{n-1} \tau_{n-1}} \alpha_{n-1}\left(\bar{x}_{n-1}\right) x_{n-1}\left(t-\tau_{n-1}\right),
\end{aligned}\right.
$$

where, for $i \in I_{n-1}, \alpha_{i}(x)=d\left(x \beta_{i}(x)\right) / d x$. One can notice that we have used the notation $x_{i}$ instead of $x_{i}-\bar{x}_{i}$ in (4.12), for the sake of simplicity.

System (4.12) can be written on the matrix form

$$
x^{\prime}(t)=\mathcal{A}_{0} x(t)+\sum_{i=1}^{n} \mathcal{A}_{i} x\left(t-\tau_{i}\right),
$$

where $x(t)=\left(x_{1}(t), \ldots, x_{n}(t)\right)^{\mathrm{T}}, \mathcal{A}_{0}=\operatorname{Diag}\left(\left(-\left(\delta_{j}+\alpha_{j}\left(\bar{x}_{j}\right)\right)\right)_{1 \leq j \leq n}\right)$, with $\alpha_{n}\left(\bar{x}_{n}\right)=0$, and the $(n \times n)$ matrix $\mathcal{A}_{i}, i \in I_{n}$, are defined by

$$
\begin{gathered}
\left(\mathcal{A}_{1}\right)_{j, k}= \begin{cases}2\left(1-K_{1}\right) e^{-\gamma_{1} \tau_{1}} \alpha_{1}\left(\bar{x}_{1}\right), & \text { if } j=k=1, \\
0, & \text { otherwise, }\end{cases} \\
\left(\mathcal{A}_{i}\right)_{j, k}=\left\{\begin{array}{ll}
2 K_{i-1} e^{-\gamma_{i-1} \tau_{i-1}} \alpha_{i-1}\left(\bar{x}_{i-1}\right), & \text { if } j=i \text { and } k=i-1, \\
2\left(1-K_{i}\right) e^{-\gamma_{i} \tau_{i}} \alpha_{i}\left(\bar{x}_{i}\right), & \text { if } j=k=i, \\
0, & \text { otherwise, }
\end{array} \text { for } i=2, \ldots, n-1,\right.
\end{gathered}
$$

and

$$
\left(\mathcal{A}_{n}\right)_{j, k}= \begin{cases}2 K_{n-1} e^{-\gamma_{n-1} \tau_{n-1}} \alpha_{n-1}\left(\bar{x}_{n-1}\right), & \text { if } j=n \text { and } k=n-1 \\ 0, & \text { otherwise }\end{cases}
$$


The characteristic equation of system (4.12) is then defined by

$$
\operatorname{det}\left(\lambda I d-\mathcal{A}_{0}-\sum_{i=1}^{n} e^{-\lambda \tau_{i}} \mathcal{A}_{i}\right)=0,
$$

where $I d$ denotes the identity matrix of $\mathcal{M}_{n}(\mathbb{R})$.

After easy computations, the characteristic equation reduces to

$$
\left(\lambda+\delta_{n}\right) \prod_{i=1}^{n-1}\left[\lambda+\delta_{i}+\alpha_{i}\left(\bar{x}_{i}\right)-2\left(1-K_{i}\right) e^{-\gamma_{i} \tau_{i}} \alpha_{i}\left(\bar{x}_{i}\right) e^{-\lambda \tau_{i}}\right]=0 .
$$

We recall that a steady state $\bar{E}$ of (2.1) is locally asymptotically stable if all characteristic roots of (4.12) have negative real parts, and unstable if a characteristic root with positive real part exists. The stability of the steady state can be lost only if pure imaginary roots appear.

From (4.13), characteristic roots are $\lambda=-\delta_{n}<0$ and all $\lambda$ solutions of equations

$$
\lambda+A_{i}\left(\tau_{1}, \ldots, \tau_{i}\right)-B_{i}\left(\tau_{1}, \ldots, \tau_{i}\right) e^{-\lambda \tau_{i}}=0,
$$

for $i \in I_{n-1}$, where

$$
\begin{aligned}
& A_{i}\left(\tau_{1}, \ldots, \tau_{i}\right):=\delta_{i}+\alpha_{i}\left(\bar{x}_{i}\right), \\
& B_{i}\left(\tau_{1}, \ldots, \tau_{i}\right):=2\left(1-K_{i}\right) e^{-\gamma_{i} \tau_{i}} \alpha_{i}\left(\bar{x}_{i}\right) .
\end{aligned}
$$

We want to point out that the coefficients $A_{i}$ and $B_{i}$ depend, implicitly, on all the time delays $\tau_{j}$, with $1 \leq j \leq i$, through the values of the equilibria $\bar{x}_{i}$ (see Proposition 4 and Remark 6).

In the next paragraph, we concentrate ourselves on the stability of trivial equilibria, that is all equilibria $\bar{E}$ with at least one zero component: $E^{0}$ and $E_{j}$ with $1 \leq j \leq i_{0}-2$ (see Proposition 4).

\section{Global Asymptotic Stability of $E^{0}$ and Instability of the Equilibria $E_{j}$}

We first deal with the stability of $E^{0}$. So we suppose that $\bar{E}=E^{0}$. Hence, $\bar{x}_{i}=0$ for all $i \in I_{n}$. Since $\alpha_{i}(0)=\beta_{i}(0)$, the characteristic equations in (4.14) reduce to

$$
\lambda+\delta_{i}+\beta_{i}(0)-2\left(1-K_{i}\right) e^{-\gamma_{i} \tau_{i}} \beta_{i}(0) e^{-\lambda \tau_{i}}=0, \quad i \in I_{n-1} .
$$

Setting $z=\tau_{i} \lambda$, it is easy to check that (5.16) is equivalent to

$$
\left(z+a_{i}\right) e^{z}-b_{i}=0, \quad i \in I_{n-1},
$$

where

$$
a_{i}:=\left(\delta_{i}+\beta_{i}(0)\right) \tau_{i} \quad \text { and } \quad b_{i}=2 \tau_{i}\left(1-K_{i}\right) e^{-\gamma_{i} \tau_{i}} \beta_{i}(0)
$$


Hayes [16], in 1950, gave necessary and sufficient conditions for the roots of (5.17) to have negative real parts:

$$
a_{i}>-1, \quad a_{i}-b_{i}>0 \quad \text { and } \quad b_{i}<\eta_{i} \sin \eta_{i}-a_{i} \cos \eta_{i},
$$

where $\eta_{i}$ is the unique solution of

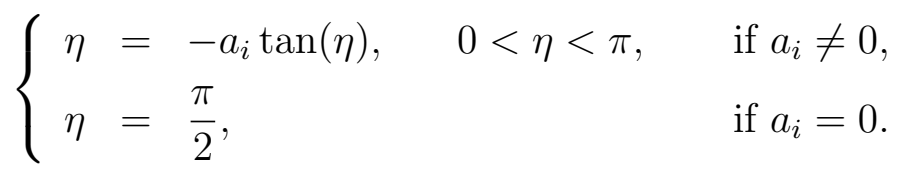

One can notice that the condition $a_{i}>-1$ in (5.18) is always satisfied since $a_{i}>0$. Moreover,

$$
a_{i}-b_{i}=\tau_{i}\left(\delta_{i}-\left(2\left(1-K_{i}\right) e^{-\gamma_{i} \tau_{i}}-1\right) \beta_{i}(0)\right)=-\tau_{i} \xi_{i}(0) .
$$

Hence, $a_{i}-b_{i}>0$ if and only if $\xi_{i}(0)<0$.

Lemma 7. Roots of (5.16) have negative real parts if and only if $\xi_{i}(0)<0$.

Proof. First, note that if $\xi_{i}(0) \geq 0$ then condition $a_{i}-b_{i}>0$ in (5.18) no longer holds and equation (5.16) has roots with nonnegative real parts. Indeed, it is easy to see that, considering the mapping $\zeta: \lambda \mapsto \lambda+\delta_{i}+\beta_{i}(0)-2\left(1-K_{i}\right) e^{-\gamma_{i} \tau_{i}} \beta_{i}(0) e^{-\lambda \tau_{i}}$ as a function of real $\lambda$, then $\zeta$ is an increasing function which tends to $+\infty$ as $\lambda$ tends to $+\infty$, with $\zeta(0) \leq 0$, since $\xi_{i}(0) \geq 0$. Consequently, with this latter assumption, equation (5.16) has always a nonnegative real root.

Assume that $\xi_{i}(0)<0$. Then $a_{i}>-1$ and $a_{i}-b_{i}>0$. Let us check that the condition $b_{i}<\eta_{i} \sin \eta_{i}-a_{i} \cos \eta_{i}$ is fulfilled.

Suppose, by contradiction, that $b_{i} \geq \eta_{i} \sin \eta_{i}-a_{i} \cos \eta_{i}$. Then, from the definition of $\eta_{i}$, we get

$$
b_{i} \geq-a_{i} \frac{1}{\cos \eta_{i}}
$$

Since $a_{i}>b_{i}$ and $a_{i}>0$, we deduce

$$
1>-\frac{1}{\cos \eta_{i}}
$$

This yields that $\cos \left(\eta_{i}\right)>0$, and consequently $\eta_{i} \in(0, \pi / 2)$. It follows that $\tan \left(\eta_{i}\right)>0$ and

$$
\eta_{i}>0>-a_{i} \tan \left(\eta_{i}\right)
$$

This contradicts the definition of $\eta_{i}$, so we conclude that $b_{i}<\eta_{i} \sin \eta_{i}-a_{i} \cos \eta_{i}$. This ends the proof.

Lemma 7 allows us to conclude, in the next proposition, to the local asymptotic stability of the equilibrium $E^{0}$. 
Proposition 8. The trivial equilibrium $E^{0}$ is locally asymptotically stable if (3.8) holds true, that is if it is the unique equilibrium of system (2.1), and unstable when (3.9) is fulfilled.

Proof. Note that $(3.8)$ is equivalent to $\xi_{1}(0)<0$, and that we have already noticed that if $\xi_{1}(0)<0$ then $\xi_{i}(0)<0$ for $i \geq 1$. Thus, with (3.8), $\xi_{i}(0)<0$ for all $i \in I_{n-1}$ and, from Lemma 7, all roots of (5.16) have negative real parts. It follows that $E^{0}$ is locally asymptotically stable.

If (3.9) holds true, then $\xi_{1}(0)>0$ and, from Lemma 7 , the characteristic equation associated with $E^{0}$ has at least one positive real root. Consequently $E^{0}$ is unstable.

Remark 9. When $\xi_{1}(0)=0$, then $\lambda=0$ is a root of (5.16) and we cannot conclude to the stability or instability of $E^{0}$ without further analysis.

In the next theorem, we establish the global asymptotic stability of $E^{0}$ for a condition stronger than (3.8).

Theorem 10. The trivial equilibrium $E^{0}$ is globally asymptotically stable if

$$
\left(2 e^{-\gamma_{1} \tau_{1}}-1\right) \beta_{1}(0)<\delta_{1}
$$

Proof. First note that, from (H1) and (H3), we have

$$
\left(2 e^{-\gamma_{i+1} \tau_{i+1}}-1\right) \beta_{i+1}(0) \leq\left(2 e^{-\gamma_{i} \tau_{i}}-1\right) \beta_{i}(0)<\delta_{i} \leq \delta_{i+1}, \quad \text { for all } i \in I_{n-1},
$$

so (5.19) implies that

$$
\left(2 e^{-\gamma_{i} \tau_{i}}-1\right) \beta_{i}(0)<\delta_{i}, \quad \text { for } i \in I_{n-1} .
$$

Now, let us denote by $C_{i}$ the set of continuous functions mapping from $\left[-\tau_{i}, 0\right]$ into $\mathbb{R}^{+}$, and let $C:=\prod_{i=1}^{n} C_{i}$. We define, for $\psi=\left(\psi_{1}, \ldots, \psi_{n}\right) \in C$,

$$
V(\psi)=\sum_{i=1}^{n} \psi_{i}(0)+\sum_{i=1}^{n-1} 2 e^{-\gamma_{i} \tau_{i}} \int_{-\tau_{i}}^{0} \beta_{i}\left(\psi_{i}(\theta)\right) \psi_{i}(\theta) d \theta
$$

The derivative of $V$ along the solutions of (2.1) is then given by

$$
\dot{V}(\psi)=\sum_{i=1}^{n} \psi_{i}^{\prime}(0)+\sum_{i=1}^{n-1} 2 e^{-\gamma_{i} \tau_{i}}\left(\beta_{i}\left(\psi_{i}(0)\right) \psi_{i}(0)-\beta_{i}\left(\psi_{i}\left(-\tau_{i}\right)\right) \psi_{i}\left(-\tau_{i}\right)\right) .
$$

Using (2.1),

$$
\begin{aligned}
\psi_{i}^{\prime}(0)= & -\left[\delta_{i}+\beta_{i}\left(\psi_{i}(0)\right)\right] \psi_{i}(0)+2\left(1-K_{i}\right) e^{-\gamma_{i} \tau_{i}} \beta_{i}\left(\psi_{i}\left(-\tau_{i}\right)\right) \psi_{i}\left(-\tau_{i}\right) \\
& +2 K_{i-1} e^{-\gamma_{i-1} \tau_{i-1}} \beta_{i-1}\left(\psi_{i-1}\left(-\tau_{i-1}\right)\right) \psi_{i-1}\left(-\tau_{i-1}\right),
\end{aligned}
$$


and one obtains

$$
\sum_{i=1}^{n} \psi_{i}^{\prime}(0)=-\delta_{n} \psi_{n}(0)+\sum_{i=1}^{n-1}\left[-\delta_{i}-\beta_{i}\left(\psi_{i}(0)\right)\right] \psi_{i}(0)+2 e^{-\gamma_{i} \tau_{i}} \beta_{i}\left(\psi_{i}\left(-\tau_{i}\right)\right) \psi_{i}\left(-\tau_{i}\right) .
$$

Hence

$$
\dot{V}(\psi)=-\delta_{n} \psi_{n}(0)+\sum_{i=1}^{n-1}\left[\left(2 e^{-\gamma_{i} \tau_{i}}-1\right) \beta_{i}\left(\psi_{i}(0)\right)-\delta_{i}\right] \psi_{i}(0) .
$$

Under assumption (5.19), we have already noticed that $\left(2 e^{-\gamma_{i} \tau_{i}}-1\right) \beta_{i}(0)<\delta_{i}$. Moreover, $\beta_{i}$ is decreasing so, since $\psi_{i}$ is nonnegative, $\beta_{i}\left(\psi_{i}(0)\right) \leq \beta_{i}(0)$. Thus, $\dot{V}(\psi) \leq 0$ for $\psi \in C$, and $V$ is a Lyapunov function on $C$ (see Hale and Verduyn Lunel [14]).

Furthermore, $\dot{V}(\psi)=0$ is equivalent to $\psi_{i}(0)=0$, for all $i \in I_{n}$, which implies, by invariance, that all solutions $x_{i}(t)$ tend to zero. It follows that all solutions of $(2.1)$ converge toward $E^{0}$ when (5.19) holds true. This concludes the proof.

We focus now on the equilibria $E_{j}, 1 \leq j \leq i_{0}-2$, defined in Proposition 4 . We suppose that (3.9) holds, that is $\xi_{1}(0)>0$, and that $i_{0} \geq 3$, so $E_{i_{0}-2} \neq E^{*}$. Since $\xi_{1}(0)>0$, then the characteristic equation (4.13) associated with $E_{j}$ has at least one positive real root (see the proof of Lemma 7). Consequently, $E_{j}$ is unstable. The next proposition is straightforward.

Proposition 11. All equilibria $E_{j}$, with $1 \leq j \leq i_{0}-2$ and $i_{0} \geq 3$, defined in Proposition 4, are unstable.

In the next section, we concentrate on the asymptotic stability of the unique positive equilibrium $E^{*}$ of system (2.1).

\section{Asymptotic Stability of the Positive Equilibrium}

We focus, in this section, on the asymptotic stability of the unique positive equilibrium of system (2.1), namely $E^{*}$. We assume that condition (3.9) holds, or equivalently that $i_{0} \geq 2$ (see Proposition 4), that is $\xi_{1}(0)>0$. From Proposition $4, E^{*}$ is then well and uniquely defined as $E^{*}=\left(x_{1}^{*}, \ldots, x_{n}^{*}\right)^{\mathrm{T}}$ where

$$
\left\{\begin{array}{l}
\xi_{1}\left(x_{1}^{*}\right)=0, \\
\xi_{i}\left(x_{i}^{*}\right)=-\frac{g_{i-1}\left(x_{i-1}^{*}\right) x_{i-1}^{*}}{x_{i}^{*}}, \quad \text { for } i=2, \ldots, n .
\end{array}\right.
$$

The functions $g_{i-1}$ and $\xi_{i}$ are respectively defined by (2.2) and (3.7). It is easy to check, from (3.9), that $\xi_{1}(0)>0$ is equivalent to

$$
\tau_{1} \in\left[0, \frac{1}{\gamma_{1}} \ln \left(\frac{2\left(1-K_{1}\right) \beta_{1}(0)}{\delta_{1}+\beta_{1}(0)}\right)\right) \quad \text { and } \quad\left(1-2 K_{1}\right) \beta_{1}(0)>\delta_{1} .
$$


In particular, $1-2 K_{1}>0$.

In order to analyze the asymptotic stability of $E^{*}$, we proceed as follows. First, we assume that $\tau_{i}=0$ for all $i \in I_{n-1}$, and show that $E^{*}$ is then locally asymptotically stable (see Lemma 13). Second, we let $\tau_{i}=0$ for all $i \in I_{n-2}$ and find a maximal range for $\tau_{n-1}$, say $\left[0, \tau_{n-1}^{*}\right)$, such that $E^{*}$ is locally asymptotically stable for all $\tau_{n-1} \in\left[0, \tau_{n-1}^{*}\right)$. The next step consists in letting $\tau_{i}=0$ for all $i \in I_{n-3}$, taking $\tau_{n-2}<\tau_{n-1}$, and applying the same procedure as the one used for $\tau_{n-1}$. And so on until we find a maximal range for $\tau_{1}$.

Remark 12. The functions $g_{i}, i \in I_{n-1}$, defined in (2.2) by

$$
g_{i}(y)=2 K_{i} e^{-\gamma_{i} \tau_{i}} \beta_{i}(y)
$$

depend explicitly on $\tau_{i}$. Moreover, these functions are always evaluated, in the following, at the steady state values $x_{i}^{*}$ which depend (see Remark 6 ) on $\tau_{1}, \ldots, \tau_{i}$. Hence, $g_{i}\left(x_{i}^{*}\right)$ is a value depending, explicitly or implicitly, on all values $\tau_{j}, 1 \leq j \leq i$. In the following, we will only write the dependence of $g_{i}$ on $\tau_{i}, g_{i}\left(\tau_{i}, x_{i}^{*}\right)$.

We first assume that $\tau_{i}=0$ for all $i \in I_{n-1}$. Then, the characteristic equation (4.13) becomes

$$
\left(\lambda+\delta_{n}\right) \prod_{i=1}^{n-1}\left[\lambda+A_{i}-B_{i}\right]=0,
$$

where $A_{i}$ and $B_{i}$ are defined in (4.15), that is in this case

$$
\begin{aligned}
& A_{i}=\delta_{i}+\beta_{i}\left(x_{i}^{*}\right)+x_{i}^{*} \beta_{i}^{\prime}\left(x_{i}^{*}\right), \\
& B_{i}=2\left(1-K_{i}\right)\left[\beta_{i}\left(x_{i}^{*}\right)+x_{i}^{*} \beta_{i}^{\prime}\left(x_{i}^{*}\right)\right] .
\end{aligned}
$$

We deduce that (6.20) has $n$ roots, denoted by $\lambda_{i}$ and given by

$$
\begin{aligned}
\lambda_{1} & =\left(1-2 K_{1}\right) x_{1}^{*} \beta_{1}^{\prime}\left(x_{1}^{*}\right)<0, \\
\lambda_{i} & =\left(1-2 K_{i}\right) x_{i}^{*} \beta_{i}^{\prime}\left(x_{i}^{*}\right)-\frac{g_{i-1}\left(0, x_{i-1}^{*}\right)}{x_{i}^{*}} x_{i-1}^{*}, \quad i=2, \ldots, n-1, \\
\lambda_{n} & =-\delta_{n}<0 .
\end{aligned}
$$

Thus, using (H2), we obtain that $\lambda_{i}<0, i \in I_{n-1}$, if $K_{n-1}<\frac{1}{2}$. We state the following lemma.

Lemma 13. Assume that $\tau_{i}=0$ for $i \in I_{n-1}$. Then the positive equilibrium $E^{*}$ of (2.1) is locally asymptotically stable if $K_{n-1}<\frac{1}{2}$.

It is admitted that, commonly, cells lose their self-renewal capacity after a consequent number of divisions, so the $K_{i}$ coefficients should approach 1 as $i$ approaches $n-1$. Hence, the above assumption seems to be not biologically realistic. However, notice here that it is a 
sufficient condition for the local asymptotic stability and not a necessary one. It is possible that more realistic assumptions lead to the local asymptotic stability but they would involve to add hypothesis on the links between $\beta_{i}$ and $\beta_{i-1}$ not easy to handle with.

We assume $\tau_{i}=0$ for $i \in I_{n-2}$ and $K_{n-1}<1 / 2$. We are going to fix an interval $\left[0, \tau_{n-1}^{\max }\right]$ for $\tau_{n-1}$, and search for $\tau_{n-1}^{*} \in\left[0, \tau_{n-1}^{\max }\right]$ such that pure imaginary roots $\pm i \omega$ appear. We prove the next lemma.

Lemma 14. Assume $K_{n-1}<1 / 2$ and $\tau_{n-1} \in\left[0, \tau_{n-1}^{\max }\right]$, where

$$
\tau_{n-1}^{\max }:=\frac{1}{\gamma_{n-1}} \ln \left[2\left(1-K_{n-1}\right)\right] .
$$

Then 0 is not an eigenvalue of (4.12). Moreover, if $i \omega, \omega \in \mathbb{R}$, is an eigenvalue, then so is $-i \omega$.

Proof. Let us assume that $\lambda=0$ is a solution of

$$
\lambda+A_{n-1}\left(\tau_{n-1}\right)-B_{n-1}\left(\tau_{n-1}\right) e^{-\lambda \tau_{n-1}}=0 .
$$

Then $A_{n-1}\left(\tau_{n-1}\right)=B_{n-1}\left(\tau_{n-1}\right)$, which is equivalent to

$$
\delta_{n-1}=\left[2\left(1-K_{n-1}\right) e^{-\gamma_{n-1} \tau_{n-1}}-1\right] \alpha_{n-1}\left(x_{n-1}^{*}\right),
$$

where we recall that $\alpha_{n-1}(x)=\frac{d}{d x}\left(x \beta_{n-1}(x)\right)$.

Using (3.5) and the fact that $\tau_{i}=0$ for $i \in I_{n-2}$, then (6.22) becomes

$$
-\frac{g_{n-2}\left(0, x_{n-2}^{*}\right) x_{n-2}^{*}}{x_{n-1}^{*}}+x_{n-1}^{*} \beta_{n-1}^{\prime}\left(x_{n-1}^{*}\right)\left[2\left(1-K_{n-1}\right) e^{-\gamma_{n-1} \tau_{n-1}}-1\right]=0 .
$$

Since $g_{n-2}\left(0, x_{n-2}^{*}\right) x_{n-2}^{*}>0, \beta_{n-1}^{\prime}\left(x_{n-1}^{*}\right)<0$ and, since $\tau_{n-1} \leq \tau_{n-1}^{\max }, 2\left(1-K_{n-1}\right) e^{-\gamma_{n-1} \tau_{n-1}}-$ $1 \geq 0$, we deduce that

$$
-\frac{g_{n-2}\left(0, x_{n-2}^{*}\right) x_{n-2}^{*}}{x_{n-1}^{*}}+x_{n-1}^{*} \beta_{n-1}^{\prime}\left(x_{n-1}^{*}\right)\left[2\left(1-K_{n-1}\right) e^{-\gamma_{n-1} \tau_{n-1}}-1\right]<0 .
$$

This gives a contradiction, so $\lambda=0$ is not an eigenvalue of (4.12).

The second statement in Lemma 14 is straightforward.

In the sequel, we suppose that $\tau_{n-1} \in J_{n-1}^{\max }:=\left[0, \tau_{n-1}^{\max }\right]$. Replacing $\lambda$ in the characteristic equation (6.21) by $i \omega, \omega>0$, and separating real and imaginary parts, we obtain

$$
\left\{\begin{array}{l}
B_{n-1}\left(\tau_{n-1}\right) \cos \left(\omega \tau_{n-1}\right)=A_{n-1}\left(\tau_{n-1}\right) \\
B_{n-1}\left(\tau_{n-1}\right) \sin \left(\omega \tau_{n-1}\right)=-\omega
\end{array}\right.
$$

Thus, adding the squares of both sides of system (6.23), we deduce that necessarily

$$
\omega^{2}=B_{n-1}^{2}\left(\tau_{n-1}\right)-A_{n-1}^{2}\left(\tau_{n-1}\right),
$$


where $A_{n-1}\left(\tau_{n-1}\right)$ and $B_{n-1}\left(\tau_{n-1}\right)$ are given in $(4.15)$, with $\tau_{1}=\cdots=\tau_{n-2}=0$.

Our aim is to determine values of $\tau_{n-1}$ such that $B_{n-1}^{2}\left(\tau_{n-1}\right)-A_{n-1}^{2}\left(\tau_{n-1}\right)$ is positive (we recall that $\lambda=0$ is not a characteristic root). We have already noticed, in the proof of Lemma 14, that $B_{n-1}\left(\tau_{n-1}\right)-A_{n-1}\left(\tau_{n-1}\right)<0$ so we focus, in the following, on the quantity $B_{n-1}\left(\tau_{n-1}\right)+A_{n-1}\left(\tau_{n-1}\right)$, and we search for conditions giving $B_{n-1}\left(\tau_{n-1}\right)+A_{n-1}\left(\tau_{n-1}\right)<0$ for some values of $\tau_{n-1} \in J_{n-1}^{\max }$.

First, let check that

$$
B_{n-1}\left(\tau_{n-1}\right)+A_{n-1}\left(\tau_{n-1}\right)=\left[2\left(1-K_{n-1}\right) e^{-\gamma_{n-1} \tau_{n-1}}+1\right] \alpha_{n-1}\left(x_{n-1}^{*}\left(\tau_{n-1}\right)\right)+\delta_{n-1} .
$$

Therefore

$$
B_{n-1}(0)+A_{n-1}(0)=\left(3-2 K_{n-1}\right) \alpha_{n-1}\left(x_{n-1}^{*}(0)\right)+\delta_{n-1} .
$$

We set

$$
\chi_{n-1}:=\frac{g_{n-2}\left(0, x_{n-2}^{*}\right) x_{n-2}^{*}}{\delta_{n-1}},
$$

and we denote by $\pi_{n-1}$ the unique solution of

$$
\left(1-2 K_{n-1}\right) \beta_{n-1}(\pi)=\delta_{n-1}-\frac{g_{n-2}\left(0, x_{n-2}^{*}\right) x_{n-2}^{*}}{\pi} .
$$

We then state and prove the following lemma.

Lemma 15. Assume $\alpha_{n-1}$ is decreasing on $\left(\chi_{n-1}, \pi_{n-1}\right)$, with

$$
-\frac{\delta_{n-1}}{2}<\alpha_{n-1}\left(\chi_{n-1}\right) \quad \text { and } \quad \alpha_{n-1}\left(\pi_{n-1}\right)<-\frac{\delta_{n-1}}{3-2 K_{n-1}} .
$$

Then there exists a unique $\tau_{n-1}^{*}$ such that

$$
\left\{\tau_{n-1} \in J_{n-1}^{\max } ; B_{n-1}\left(\tau_{n-1}\right)+A_{n-1}\left(\tau_{n-1}\right)<0\right\}=\left[0, \tau_{n-1}^{*}\right) .
$$

Proof. First notice that the mapping $\tau_{n-1} \in J_{n-1}^{\max } \mapsto x_{n-1}^{*}\left(\tau_{n-1}\right)$ is decreasing, with

$$
x_{n-1}^{*}(0)=\pi_{n-1} \quad \text { and } \quad x_{n-1}^{*}\left(\tau_{n-1}^{\max }\right)=\chi_{n-1} .
$$

With the assumptions on $\alpha_{n-1}$ it follows that the mapping $\tau_{n-1} \in J_{n-1}^{\max } \mapsto \alpha_{n-1}\left(x_{n-1}^{*}\left(\tau_{n-1}\right)\right)$ is negative and increasing.

Since $\tau_{n-1} \in J_{n-1}^{\max } \mapsto 2\left(1-K_{n-1}\right) e^{-\gamma_{n-1} \tau_{n-1}}+1$ is positive and decreasing, we deduce that the mapping $\tau_{n-1} \in J_{n-1}^{\max } \mapsto B_{n-1}\left(\tau_{n-1}\right)+A_{n-1}\left(\tau_{n-1}\right)$ is increasing with

$$
B_{n-1}(0)+A_{n-1}(0)=\left(3-2 K_{n-1}\right) \alpha_{n-1}\left(\pi_{n-1}\right)+\delta_{n-1}<0
$$

and

$$
B_{n-1}\left(\tau_{n-1}^{\max }\right)+A_{n-1}\left(\tau_{n-1}^{\max }\right)=2 \alpha_{n-1}\left(\chi_{n-1}\right)+\delta_{n-1}>0 .
$$

Existence and uniqueness of $\tau_{n-1}^{*}$ follow. 
In the sequel, we suppose that there exists an interval $\left[0, \tau_{n-1}^{*}\right) \subset J_{n-1}^{\max }$ such that $B_{n-1}^{2}\left(\tau_{n-1}\right)-A_{n-1}^{2}\left(\tau_{n-1}\right)>0$ for $\tau_{n-1} \in\left[0, \tau_{n-1}^{*}\right)$. Then we deduce from the first equation in (6.23) that

$$
\tau_{n-1}=\frac{\arccos \left(\frac{A_{n-1}\left(\tau_{n-1}\right)}{B_{n-1}\left(\tau_{n-1}\right)}\right)+2 k \pi}{\sqrt{B_{n-1}^{2}\left(\tau_{n-1}\right)-A_{n-1}^{2}\left(\tau_{n-1}\right)}}, \quad k \in \mathbb{N} .
$$

Therefore, critical values of $\tau_{n-1}$ for which a pair of pure imaginary characteristic roots appear are zeros of the functions

$$
Z_{n-1}^{k}(\tau)=\tau-\frac{\arccos \left(\frac{A_{n-1}(\tau)}{B_{n-1}(\tau)}\right)+2 k \pi}{\sqrt{B_{n-1}^{2}(\tau)-A_{n-1}^{2}(\tau)}}, \quad k \in \mathbb{N}, \quad \tau \in\left[0, \tau_{n-1}^{*}\right) .
$$

From Beretta and Kuang [5], we know that each pair of pure imaginary characteristic roots $\pm i \omega\left(\tau_{n-1}^{c}\right)$, with $Z_{n-1}^{k}\left(\tau_{n-1}^{c}\right)=0$ for some $k \in \mathbb{N}$, is simple and

$$
\left.\operatorname{sign}\left\{\left.\frac{d \operatorname{Re}(\lambda(\tau))}{d \tau}\right|_{\tau=\tau_{n-1}^{c}}\right\}=\operatorname{sign}\left\{2 \omega\left(\tau_{n-1}^{c}\right)\right)\right\} \operatorname{sign}\left\{\left.\frac{d Z_{n-1}^{k}(\tau)}{d \tau}\right|_{\tau=\tau_{n-1}^{c}}\right\},
$$

which simplifies here in

$$
\operatorname{sign}\left\{\left.\frac{d \operatorname{Re}(\lambda(\tau))}{d \tau}\right|_{\tau=\tau_{n-1}^{c}}\right\}=\operatorname{sign}\left\{\left.\frac{d Z_{n-1}^{k}(\tau)}{d \tau}\right|_{\tau=\tau_{n-1}^{c}}\right\} .
$$

The following theorem sums up the behavior of the positive steady-state $E^{*}$.

Theorem 16. Assume $K_{n-1}<\frac{1}{2}$ and $\tau_{j}=0$ for $j \in I_{n-2}$.

(i) If $Z_{n-1}^{0}$ has no positive root, then $E^{*}$ is locally asymptotically stable for $\tau_{n-1} \in J_{n-1}^{\max }$.

(ii) If $Z_{n-1}^{0}$ has at least one positive root, then there exists $T_{n-1} \in\left(0, \tau_{n-1}^{*}\right)$ such that $E^{*}$ is locally asymptotically stable for $\tau_{n-1} \in\left[0, T_{n-1}\right)$ and becomes unstable for $\tau_{n-1}>T_{n-1}$ (with a stability switch that can possibly occur for a larger value of $\tau_{n-1}$ ), and a Hopf bifurcation occurs at $E^{*}$ when $\tau_{n-1}=T_{n-1}$ provided that

$$
\frac{d Z_{n-1}^{0}}{d \tau}\left(T_{n-1}\right)>0
$$

We can now use a step method to obtain the existence of a critical value $T_{n-2}>0$ for which $E^{*}$ is locally asymptotically stable when $\tau_{n-2}<T_{n-2}$, and $\tau_{j}=0$ for $j \in I_{n-3}$.

To that aim, let us fix $\tau_{n-1}$ in an interval $\left[0, T_{n-1}\right)$ such that $E^{*}$ is locally asymptotically stable when $\tau_{j}=0, j \in I_{n-2}$, and $\tau_{n-1} \in\left[0, T_{n-1}\right.$ ) (Note that $T_{n-1}$ may be the critical value given by (ii) in Theorem 16, but it may also be the upper bound of $J_{n-1}^{\max }$ ). 
Then, let us fix $\tau_{j}=0$ for $j \in I_{n-3}$, and use the same reasoning as above to obtain the existence of a critical value $0<T_{n-2}\left(\tau_{n-1}\right)<\tau_{n-1}$ such that $E^{*}$ is locally asymptotically stable when $\tau_{n-1} \in\left[0, T_{n-1}\right), \tau_{n-2} \in\left[0, T_{n-2}\right)$, and $\tau_{j}=0, j \in I_{n-3}$.

This reasoning is based on the fact that the time delay $\tau_{n-1}$ only plays a role in the equations for $x_{n-1}$ and $x_{n}$, and the characteristic equation (4.13) is the product of exponential polynomial functions that do not depend on $\tau_{n-1}$, except the last one.

Using this procedure, we find a range of stability for $\tau_{1}$, say $\left[0, T_{1}\right)$, such that $E^{*}$ is locally asymptotically stable for $\tau_{n-1} \in\left[0, T_{n-1}\right), \tau_{n-2} \in\left[0, T_{n-2}\left(\tau_{n-1}\right)\right), \ldots, \tau_{2} \in\left[0, T_{2}\left(\tau_{3}\right)\right)$ and $\tau_{1} \in\left[0, T_{1}\left(\tau_{2}\right)\right)$, with $T_{i}\left(\tau_{i+1}\right)<\tau_{i+1}$. This is resumed in the next theorem.

Theorem 17. Assume $K_{n-1}<\frac{1}{2}$. Then there exists a sequence $\left(T_{i}\right)_{i \in I_{n-1}}$ such that $E^{*}$ is locally asymptotically stable for $\tau_{i} \in\left[0, T_{i}\right), i \in I_{n-1}$, where the sequence $\left(T_{i}\right)$ satisfies

$$
0<T_{i}<\tau_{i+1}<T_{i+1} \quad \text { and } \quad T_{i}=T_{i}\left(\tau_{i+1}\right), \quad i \in I_{n-2} .
$$

Let us illustrate numerically, using the MATLAB solver dde23 [25], the result in Theorem 17. We consider four sub cell populations $\left(x_{1}, x_{2}, x_{3}\right.$ and $\left.x_{4}\right)$ : the pluripotent hematopoietic stem cells, progenitor cells, precursor cells, and mature cells. Then for short cell cycle durations (less than one day), these populations are stable (see Figure 2). We want to point out that the initial conditions used in the following have the form $\left(x_{1}, 0,0,0\right)$, to describe, for example, a process that starts with only pluripotent hematopoietic stem cells. Moreover, the nonlinear introduction functions $\beta_{i}$ are defined by

$$
\beta_{i}(x)=\frac{\beta_{i}^{0}}{1+x^{3}}, \quad \text { with } \beta_{i}^{0}>0
$$

One interesting feature, is to observe, numerically, that oscillations may appear in differentiated cell lines, even though the pluripotent hematopoietic stem cell population is stable (see Figure 3).

Using the model in [19], Pujo-Menjouet and Mackey [24] showed that long period oscillations could be observed within the hematopoietic stem cell compartment and be associated with hematological diseases [15], such as neutropenias and leukemias, which can sometimes exhibit oscillations of all blood cell counts and whose origins lie in the pluripotent hematopoietic stem cell population. See, on this topic, the various works of Fortin and Mackey [13], Bernard et al. [6, 7], Pujo-Menjouet et al. [23], Adimy et al. [2], and the references therein.

The fact that oscillations of circulating blood cells can occur without oscillations of pluripotent hematopoietic stem cells has never been mathematically investigated (the main reason is probably the complexity of models taking into account all the major events in the blood production process). It seems that our model is able to produce such behaviors, and then could give a new insight on the origin and the reasons of periodic hematological diseases [15]. This is a point that would deserve more attention and, since this was not the objective of this work, we let it for future analysis. 


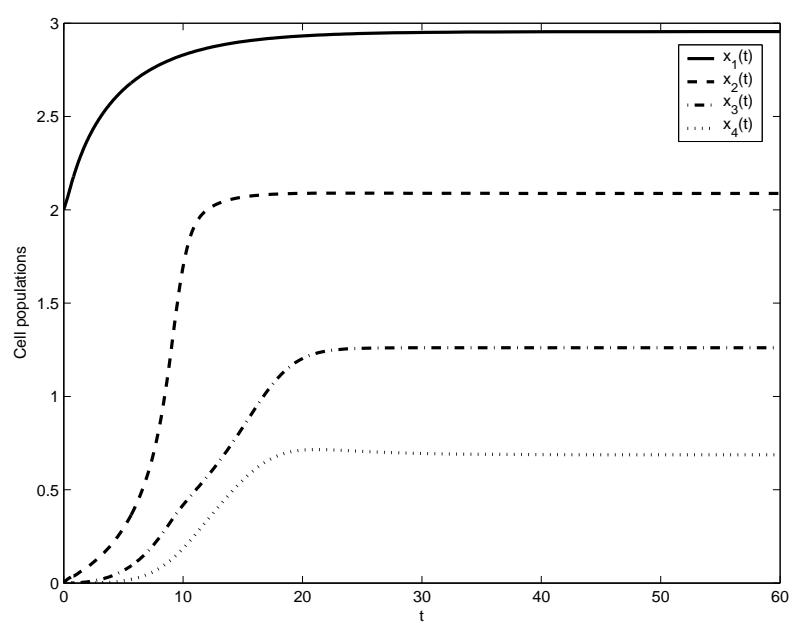

Figure 2: Four sub populations are considered: the pluripotent hematopoietic stem cell population $x_{1}$ (solid line), the progenitor cell population $x_{2}$ (dashed line), the precursor cell population $x_{3}$ (dash-dotted line), and the mature cell population $x_{4}$ (dotted line). With an initial condition in the form $\left(x_{1}, 0,0,0\right)$, describing a process starting only with pluripotent hematopoietic stem cells, the cell populations are stable and converge toward the unique nontrivial steady state of the system. Parameters values: $\delta_{1}=0.05, \delta_{2}=0.1, \delta_{3}=0.15$, $\delta_{4}=0.2, \beta_{1}^{0}=2, \beta_{2}^{0}=1.5, \beta_{3}^{0}=1, \gamma_{1}=0.15, \gamma_{2}=0.18, \gamma_{3}=0.2, K_{1}=0.1, K_{2}=0.11$, $K_{3}=0.2$, and $\tau_{1}=0.5, \tau_{2}=0.8, \tau_{3}=1$.

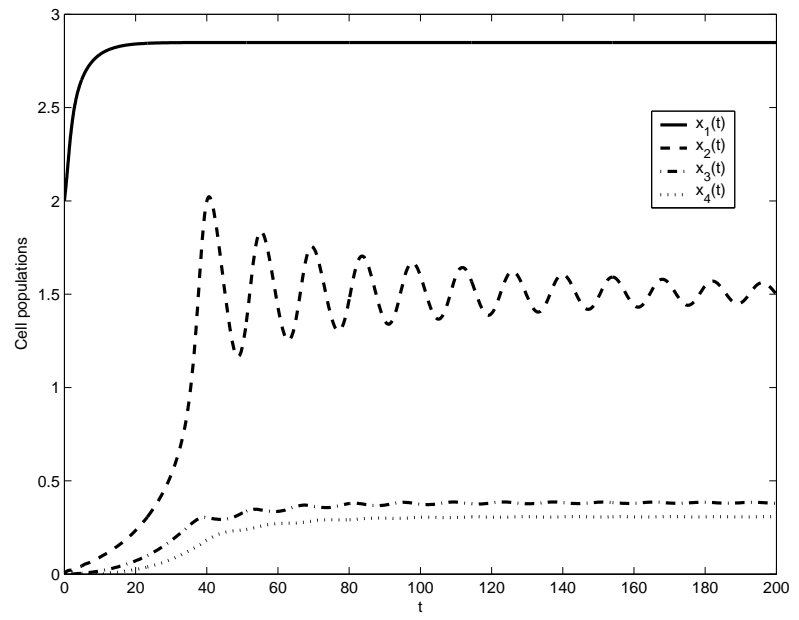

Figure 3: As in Figure 2, four sub populations are considered, with an initial condition in the form $\left(x_{1}, 0,0,0\right)$. The cell populations are stable, but oscillations are observed for $x_{2}$, $x_{3}$ and $x_{4}$ (even though the amplitudes of the oscillations for $x_{3}$ and $x_{4}$ are rather small) whereas $x_{1}$ does not oscillate and converge toward the equilibrium. Parameters values are the same than in Figure 2, except $\gamma_{1}=0.17, \gamma_{2}=0.18, \gamma_{3}=0.19, K_{1}=0.05, K_{2}=0.1$, $K_{3}=0.15$, and $\tau_{1}=1, \tau_{2}=2, \tau_{3}=3$. 


\section{References}

[1] M. Adimy, F. Crauste. Global stability of a partial differential equation with distributed delay due to cellular replication. Nonlinear Analysis, 54 (2003), No. 8, 1469-1491.

[2] M. Adimy, F. Crauste, S. Ruan. A mathematical study of the hematopoiesis process with applications to chronic myelogenous leukemia. SIAM J. Appl. Math., 65 (2005), No. 4, 1328-1352.

[3] M. Adimy, F. Crauste, S. Ruan. Stability and Hopf bifurcation in a mathematical model of pluripotent stem cell dynamics. Nonlinear Analysis: Real World Applications, 6 (2005), No. 4, 651-670.

[4] M. Adimy, L. Pujo-Menjouet. Asymptotic behaviour of a singular transport equation modelling cell division. Discret. Cont. Dyn. Sys. Ser. B, 3 (2003), 439-456.

[5] E. Beretta, Y. Kuang. Geometric stability switch criteria in delay differential systems with delay dependent parameters, SIAM J. Math. Anal., 33 (2002), No. 5, 1144-1165.

[6] S. Bernard, J. Bélair, M.C. Mackey. Oscillations in cyclical neutropenia: New evidence based on mathematical modeling. J. Theor. Biol., 223 (2003), 283-298.

[7] S. Bernard, J. Bélair, M.C. Mackey. Bifurcations in a white-blood-cell production model. C. R. Biologies, 327 (2004), 201-210.

[8] S. Bernard, L. Pujo-Menjouet, M.C. Mackey. Analysis of cell kinetics using a cell division marker: Mathematical analysis of experimental data, Biophy. J., 84 (2003), 34143424.

[9] F.J. Burns, I.F. Tannock. On the existence of a $G_{0}$ phase in the cell cycle. Cell Tissue Kinet., 19 (1970), 321-334.

[10] J. Dyson, R. Villella-Bressan, G.F. Webb. A singular transport equation modelling a proliferating maturity structured cell population. Can. Appl. Math. Quart., 4 (1996), 65-95.

[11] J. Dyson, R. Villella-Bressan, G.F. Webb. A nonlinear age and maturity structured model of population dynamics, I: Basic theory. J. Math. Anal. Appl., 242 (2000), No. 1, 93-104.

[12] J. Dyson, R. Villella-Bressan, G.F. Webb. A nonlinear age and maturity structured model of population dynamics, II: Chaos. J. Math. Anal. Appl., 242 (2000), No. 2, 255-270.

[13] P. Fortin, M.C. Mackey. Periodic chronic myelogenous leukemia: Spectral analysis of blood cell counts and etiological implications. Brit. J. Haematol., 104 (1999), 336-345. 
[14] J. Hale, S.M. Verduyn Lunel. Introduction to functional differential equations, Applied Mathematical Sciences 99, Springer-Verlag, New York, 1993.

[15] C. Haurie, D.C. Dale, M.C. Mackey. Cyclical neutropenia and other periodic hematological diseases: A review of mechanisms and mathematical models. Blood, 92 (1998), 2629-2640.

[16] N.D. Hayes. Roots of the transcendental equation associated with a certain differential difference equation. J. London Math. Soc., 25 (1950), 226-232.

[17] P.C.L. John. The cell cycle, London, Cambridge University Press, 1981.

[18] L.G. Lajtha. On DNA labeling in the study of the dynamics of bone marrow cell populations. In: Stohlman, Jr., F. (Ed), The Kinetics of Cellular Proliferation, Grune and Stratton, New York, 1959, 173-182.

[19] M.C. Mackey. Unified hypothesis of the origin of aplastic anaemia and periodic hematopoïesis. Blood, 51 (1978), 941-956.

[20] M.C. Mackey, R. Rudnicki. Global stability in a delayed partial differential equation describing cellular replication. J. Math. Biol., 33 (1994), 89-109.

[21] M.C. Mackey, R. Rudnicki. A new criterion for the global stability of simultaneous cell replication and maturation processes. J. Math. Biol., 38 (1999), 195-219.

[22] J.M. Mitchison. The biology of the cell cycle, London, Cambridge University Press, 1971.

[23] L. Pujo-Menjouet, S. Bernard, M.C. Mackey. Long period oscillations in a $G_{0}$ model of hematopoietic stem cells. SIAM J. Appl. Dynam. Syst., 4 (2005), No. 2, 312-332.

[24] L. Pujo-Menjouet, M.C. Mackey. Contribution to the study of periodic chronic myelogenous leukemia. C. R. Biologies, 327 (2004), 235-244.

[25] L.F. Shampine, S. Thompson. Solving DDEs in MatlaB. Appl. Numer. Math., 37 (2001), 441-458. http://www.radford.edu/ thompson/webddes/. 